\title{
PARITY, AND THE MECHANICAL ENERGY OF A HEATED BODY*
}

\author{
BY \\ W. A. DAY \\ Hertford College, Oxford
}

1. Introduction. According to the isothermal theory of elasticity, the mechanical energy of an elastic body-the sum of the strain energy and the kinetic energyremains constant if the rate of working of the surface and body forces is equal to zero.

The mechanical energy, henceforth denoted by $\Phi$, ceases to be constant once interaction with the dissipative effects of heat conduction is taken into account, but exactly how it behaves depends, among other things, upon the response of the body and upon conditions at the boundary. In certain circumstances, $\Phi$ is known to decay to zero (Dafermos [1], Dassios and Grillakis [2], Day [3]).

The purpose of the present paper is to examine the behaviour of $\Phi$ when the rate of working of surface and body forces vanishes and the coupling between mechanical and thermal effects is small: the positive number $\gamma-1, \gamma$ being the ratio of the specific heat at constant stress to that at constant strain, provides an appropriate measure of the degree of coupling.

In order to gauge the behaviour of $\Phi$, we suppose $\Phi(t)$ to be defined at all times $t \geq 0$ and ask how long it takes for $\Phi$ to decay to a specified fraction, one-half say, of its initial value. (The choice of fraction has no particular significance. Similar considerations apply if $1 / 2$ is replaced by any $\varepsilon$ in $0<\varepsilon<1$ ).

To make matters precise, let us introduce the notion of the half-life $\tau$ by considering the set

$$
\left\{t: t \geq 0 \text { and } \Phi(t)=\frac{1}{2} \Phi(0)\right\} .
$$

Two possibilities can arise. The set may be empty, in which case ( $\Phi$ being continuous) $\Phi(t)>\frac{1}{2} \Phi(0)$ for every $t \geq 0$; if so we put $\tau=+\infty$. When the set is not empty, we define $\tau$ to be the greatest lower bound of the set; thus, when it is finite, $\tau$ is the first value of $t$ at which $\Phi(t)=\frac{1}{2} \Phi(0)$. In the event that $\Phi(0)=0$ the half-life $\tau=0$, but $\tau>0$ whenever $\Phi(0)>0$.

The isothermal theory, which deals only with what happens when $\gamma=1$, implies that $\tau=+\infty$ if $\Phi(0)>0$. It is tempting, therefore, to make the

Conjecture. $\tau \rightarrow+\infty$ as $\gamma \rightarrow 1$, provided $\Phi(0)>0$ and the rate of working of the surface and body forces is equal to zero.

*Received December 20, 1986. 
This conjecture is false, as we shall see by constructing a simple example. Indeed, the failure is a striking one for, given arbitrarily small positive numbers $\delta$ and $\varepsilon$, it can happen that $\gamma<1+\delta$ and $\tau<\varepsilon$.

On the other hand, we shall be able to show that in a wide, but by no means all-embracing, set of circumstances the half-life

$$
\tau \geq \frac{\text { constant }}{\gamma-1},
$$

where the constant is positive and independent of $\gamma$. Thus, the conjecture is correct in these circumstances.

The argument turns in part upon an observation about the behaviour of the equations of the linear theory of coupled dynamic thermoelasticity with respect to a geometric transformation-the parity transformation; its elementary character notwithstanding, the observation would seem not to have been exploited before.

2. Coupled thermoelasticity. We consider a finite body $B$ that is homogeneous but may be anisotropic; the body occupies a region that is bounded and regular (Gurtin [4], Sec. 15). In the absence of body force and heat supply fields, the underlying equations are the equation of motion

$$
\operatorname{div} \mathbf{S}=\rho \ddot{\mathbf{u}},
$$

the equation of energy

$$
\operatorname{div} \mathbf{q}=\rho c \dot{\varphi}+\theta_{0} \mathbf{M} \cdot \dot{\mathbf{E}},
$$

the strain-displacement relation

$$
\mathbf{E}=\operatorname{sym} \nabla \mathbf{u},
$$

the thermal gradient-temperature relation

$$
\mathbf{g}=\nabla \varphi,
$$

the stress-strain-temperature relation

$$
\mathbf{S}=\mathbf{C}[\mathbf{E}]-\varphi \mathbf{M},
$$

and the heat conduction equation

$$
\mathbf{q}=\mathbf{K g} \text {. }
$$

The positive constant $\theta_{0}$ is the uniform reference temperature, measured on the absolute scale; $\mathbf{u}$ is the displacement, $\mathbf{E}$ is the strain tensor, $\mathbf{S}$ is the stress tensor, $\varphi$ is the temperature difference (i.e., $\theta_{0}+\varphi$ is the absolute temperature), $\mathbf{g}$ is the thermal gradient, and $\mathbf{q}$ is the heat flux vector.

A list $[\mathbf{u}, \mathbf{E}, \mathbf{S}, \varphi, \mathbf{g}, \mathbf{q}]$, of fields that are appropriately smooth (in the sense of Carlson [5], Sec. 18) and satisfy the underlying equations, is said to define a thermoelastic process corresponding to zero body force and zero heat supply. The associated surface traction is defined in the usual way, i.e., as

$$
\mathbf{s}=\mathbf{S n},
$$

where $\mathbf{n}$ is the unit outward normal to the boundary $\partial B$. 
The scalars $\rho$ and $c$ are the mass density and the specific heat at constant strain, respectively; $\mathbf{C}$ is the fourth-order elasticity tensor, $\mathbf{K}$ is the conductivity tensor, and $\mathbf{M}$ is the stress-temperature tensor.

It should be kept in mind that our notation differs from that of Carlson in three minor respects: firstly, $c$ is the specific heat of unit mass at constant strain, whereas Carlson's $c$ is the specific heat of unit volume at constant strain; next, the stresstemperature tensor $\mathbf{M}$ is the negative of Carlson's and, likewise, the heat flux vector $\mathbf{q}$ is the negative of his.

It will be supposed throughout that $\rho$ and $c$ are positive, $\mathbf{C}$ is symmetric and positive definite, $\mathbf{K}$ is positive definite (but not necessarily symmetric), and $\mathbf{M}$ is symmetric.

The specific heat of unit mass at constant stress is

$$
c+\frac{\theta_{0}}{\rho} \mathbf{M} \cdot \mathbf{C}^{-1}[\mathbf{M}]
$$

and exceeds $c$; the ratio of specific heats is

$$
\gamma=1+\frac{\theta_{0}}{\rho c} \mathbf{M} \cdot \mathbf{C}^{-1}[\mathbf{M}] .
$$

If $\mu_{\min }$ and $\mu_{\max }$ are the minimum and the maximum elastic moduli, i.e., the least and the greatest of the eigenvalues of $\mathbf{C}$, considered as a linear transformation on the space of symmetric tensors,

$$
\mu_{\min } \mathbf{M} \cdot \mathbf{C}^{-1}[\mathbf{M}] \leq|\mathbf{M}|^{2} \leq \mu_{\max } \mathbf{M} \cdot \mathbf{C}^{-1}[\mathbf{M}] .
$$

Hence $|\mathbf{M}|^{2}$ is estimated by

$$
(\gamma-1) \rho c \mu_{\min } \leq \theta_{0}|\mathbf{M}|^{2} \leq(\gamma-1) \rho c \mu_{\max },
$$

and the number $\gamma-1$ does provide a measure of the degree of coupling.

Our concern is with the behaviour of the mechanical energy

$$
\Phi=\frac{1}{2} \int_{B} \mathbf{E} \cdot \mathbf{C}[\mathbf{E}] d v+\frac{1}{2} \int_{B} \rho|\dot{\mathbf{u}}|^{2} d v,
$$

the thermal energy

$$
T=\frac{1}{2 \theta_{0}} \int_{B} \rho c \varphi^{2} d v
$$

and the total energy

$$
\mathcal{U}=\Phi+T \text {. }
$$

The hypotheses on $\theta_{0}, \rho, c, \mathbf{C}$ ensure that each of these is nonnegative.

When the rate of working of the surface forces vanishes, i.e.,

$$
\int_{B} \mathbf{s} \cdot \dot{\mathbf{u}} d a=0,
$$

the rates of change of $\Phi$ and $U$ are

$$
\begin{aligned}
& \dot{\Phi}=\int_{B} \varphi \mathbf{M} \cdot \dot{\mathbf{E}} d v, \\
& \dot{\mathcal{U}}=-\frac{1}{\theta_{0}} \int_{B} \mathbf{g} \cdot \mathbf{q} d v+\frac{1}{\theta_{0}} \int_{\partial B} \varphi \mathbf{q} \cdot \mathbf{n} d a,
\end{aligned}
$$


these formulae being special cases of the theorem of power expended and the theorem of power and energy, respectively [5, Sec. 21].

If $\mathbf{M}=\mathbf{0}$ all is plain sailing, for (2.12) implies that $\dot{\Phi}=0$ and, hence, that $\Phi$ is constant, as we kıew to be the case.

In order to study what happens when $\mathbf{M} \neq \mathbf{0}$ we shall, in effect, study a family of problems, for we shall think of $\mathbf{M}$ as being variable but small, while $\theta_{0}, \rho, c, \mathbf{C}, \mathbf{K}$ are all fixed and so is the ratio

$$
\Delta=T(0) / \Phi(0)
$$

of the initial thermal energy to the initial mechanical energy.

3. An example of rapid decay. An elementary example shows that when $\mathbf{M} \neq \mathbf{0}$ the decay of $\Phi$ can be arbitrarily rapid, i.e., $\tau$ can be arbitrarily small. For the purposes of the example the material is isotropic, and the notation is the common one in which $\lambda$ and $\mu$ are the elastic moduli, $k$ is the scalar conductivity, and $m$ is the scalar stress-temperature modulus.

Proposition. Let $B$ be an isotropic ball, let $\delta$ and $\varepsilon$ be any positive numbers, and let the stress-temperature modulus satisfy

$$
0<3 \theta_{0} m^{2}<\rho c(3 \lambda+2 \mu) \delta .
$$

Then $\gamma<1+\delta$, and there is a thermoelastic process corresponding to zero body force and zero heat supply such that (i) the rate of working of the surface forces vanishes, (ii) the initial mechanical energy $\Phi(0)>0$, (iii) the half-life $\tau<\varepsilon$.

The assertion about $\gamma$ is an immediate consequence of the upper bound on $\mathrm{m}^{2}$, for

$$
\gamma=1+\frac{3 \theta_{0} m^{2}}{\rho c(3 \lambda+2 \mu)}
$$

Elimination of $\mathbf{E}, \mathbf{S}, \mathbf{g}, \mathbf{q}$ between Eqs. (2.1)-(2.6) produces the displacementtemperature equation of motion

$$
\operatorname{div} \mathbf{C}[\nabla \mathbf{u}]=\rho \ddot{u}+\operatorname{div}(\varphi \mathbf{M})
$$

and the coupled heat equation

$$
\operatorname{div}(\mathbf{K} \nabla \varphi)=\rho c \dot{\varphi}+\theta_{0} \mathbf{M} \cdot \nabla \dot{\mathbf{u}},
$$

and, in view of isotropy, these reduce to the equations

$$
\begin{aligned}
(\lambda+\mu) \nabla \operatorname{div} \mathbf{u}+\mu \Delta \mathbf{u} & =\rho \ddot{\mathbf{u}}+m \boldsymbol{\nabla} \varphi, \\
k \Delta \varphi & =\rho c \dot{\varphi}+\theta_{0} m \operatorname{div} \dot{\mathbf{u}},
\end{aligned}
$$

respectively.

Let $\nu$ be a root of the equation

$$
\nu b \cot \nu b=1,
$$

$b$ being the radius of the ball. Considerations of sign show that the cubic equation

$$
\left((\lambda+2 \mu) \nu^{2}+\rho \xi^{2}\right)\left(k \nu^{2}-\rho c \xi\right)=\theta_{0} m^{2} \nu^{2} \xi
$$


has a root $\xi$ lying in the interval

$$
\frac{k(\lambda+2 \mu) \nu^{2}}{\rho c(\lambda+2 \mu)+\theta_{0} m^{2}}<\xi<\frac{k \nu^{2}}{\rho c},
$$

and clearly we can arrange to have $2 \xi>\log (2 / \varepsilon)$ by choosing $\nu$ sufficiently large. The displacement-temperature equations and the coupled heat equation are satisfied by the radially directed displacement

$$
\mathbf{u}(\mathbf{x}, t)=\frac{u(r, t)}{r}(\mathbf{x}-\mathbf{0}),
$$

where $r=|\mathbf{x}-\mathbf{0}|$ is distance from the centre (presumed to be the origin) and the radial component is

$$
u(r, t)=\frac{\left(k \nu^{2}-\rho c \xi\right) b}{m \xi \nu^{2} \sin \nu b}\left(\frac{1}{r^{2}} \sin \nu r-\frac{\nu}{r} \cos \nu r\right) \exp (-\xi t),
$$

and by the temperature difference

$$
\varphi(\mathbf{x}, t)=\frac{\theta_{0} b \sin \nu r}{r \sin \nu b} \exp (-\xi t) .
$$

The corresponding fields $\mathbf{E}, \mathbf{S}, \mathbf{g}, \mathbf{q}$ can be determined in turn from (2.3), (2.5), (2.4), (2.6), and the list [ $\mathbf{u}, \mathbf{E}, \mathbf{S}, \varphi, \mathbf{g}, \mathbf{q}]$ so constructed is a thermoelastic process corresponding to zero body force and zero heat supply.

Since $u(b, t)=0$, the boundary of the ball is clamped and (i) is certainly satisfied. The requirement (ii) is satisfied because the strain energy and the kinetic energy are always positive. In view of the way $\xi$ has been chosen,

$$
\Phi(t)=\Phi(0) \exp (-2 \xi t)<\Phi(0) 2^{-t / \varepsilon}
$$

and, hence, $\tau<\varepsilon$, which is (iii).

4. The half-life in a special case. Although the rate of working of the surface forces vanishes in the example just constructed, the temperature difference $\varphi$ does not vanish on the boundary. It will be shown next that the vanishing of $\varphi$ on the boundary is enough to ensure the validity of the conjecture framed in the Introduction, viz $\tau \rightarrow+\infty$ as $\gamma \rightarrow 1$.

LEMMA 1. Let [u, E, S, $\varphi, \mathbf{g}, \mathbf{q}]$ be a thermoelastic process corresponding to zero body force and zero heat supply such that the rate of working of the surface forces vanishes, the initial mechanical energy $\Phi(0)>0$, and the boundary condition

$$
\left.\varphi\right|_{\partial B}=0 \quad(t \geq 0)
$$

is satisfied. Then there is a positive constant $A$, depending only upon $\mathbf{C}, c, \mathbf{K}$, such that

$$
\tau \geq \frac{A}{(\gamma-1)(1+2 \Delta)} .
$$

In order to prove the lemma we return to the formula (2.12) for $\dot{\Phi}$. Because $\mathbf{M}$ is symmetric, the integrand

$$
\varphi \mathbf{M} \cdot \dot{\mathbf{E}}=\varphi \mathbf{M} \cdot \nabla \dot{\mathbf{u}}=\operatorname{div}(\varphi \mathbf{M u})-\dot{\mathbf{u}} \cdot \mathbf{M g},
$$


and when we invoke the divergence theorem and the boundary condition (4.1) we obtain the equation

$$
0=\dot{\Phi}+\int_{B} \dot{\mathbf{u}} \cdot \mathbf{M g} d v
$$

By virtue of the bounds (2.7),

$$
\dot{\mathbf{u}} \cdot \mathbf{M g} \leq|\dot{\mathbf{u}}||\mathbf{M}||\mathbf{g}| \leq\left(\frac{(\gamma-1) \rho c \mu_{\max }}{\theta_{0}}\right)^{1 / 2}|\dot{\mathbf{u}}||\mathbf{g}|,
$$

and we can conclude that

$$
0 \leq \dot{\Phi}+\left(\frac{(\gamma-1) c \mu_{\max }}{\theta_{0}}\right)^{1 / 2}\left(\int_{B} \rho|\dot{\mathbf{u}}|^{2} d v\right)^{1 / 2}\left(\int_{B}|\mathbf{g}|^{2} d v\right)^{1 / 2}
$$

by making use of the Schwarz inequality.

In view of the definition (2.8) of the mechanical energy,

$$
\int_{B} \rho|\dot{\mathbf{u}}|^{2} d v \leq 2 \Phi
$$

and, therefore,

$$
0 \leq \dot{\Phi}+\left(\frac{2(\gamma-1) c \mu_{\max }}{\theta_{0}}\right)^{1 / 2} \Phi^{1 / 2}\left(\int_{B}|\mathbf{g}|^{2} d v\right)^{1 / 2} .
$$

Next, we note that the formula $(2.13)$ for $\dot{\mathcal{U}}$, and the boundary condition (4.1), imply that

$$
\dot{\mathcal{U}}=-\frac{1}{\theta_{0}} \int_{B} \mathbf{g} \cdot \mathbf{K} \mathbf{g} d v \leq-\frac{k_{\min }}{\theta_{0}} \int_{B}|\mathbf{g}|^{2} d v \leq 0,
$$

where the positive constant $k_{\min }$ is the least eigenvalue of $\operatorname{sym} \mathbf{K}$, the symmetric part of the conductivity tensor. Thus $U(t)$ is a decreasing function of $t$, and (4.3) implies the inequality

$$
0 \leq \dot{\Phi}+\left(\frac{2(\gamma-1) c \mu_{\max }}{k_{\min }}\right)^{1 / 2} \Phi^{1 / 2}(-\dot{\mathcal{U}})^{1 / 2} .
$$

We may suppose the half-life $\tau$ to be finite, for the conclusion (4.2) is trivially correct if $\tau=+\infty$. Since $\Phi(0)>0$ and $\tau$ is the first time at which $\Phi(\tau)=\frac{1}{2} \Phi(0)$, it must be that $\Phi(t)>0$ throughout the interval $0 \leq t \leq \tau$ and, hence, (4.4) implies

$$
0 \leq \int_{0}^{\tau} \Phi^{-1 / 2} \dot{\Phi} d t+\left(\frac{2(\gamma-1) c \mu_{\max }}{k_{\min }}\right)^{1 / 2} \int_{0}^{\tau}(-\dot{\mathcal{U}})^{1 / 2} d t .
$$

The first integral

$$
\int_{0}^{\tau} \Phi^{-1 / 2} \dot{\Phi} d t=2\left(\Phi(\tau)^{1 / 2}-\Phi(0)^{1 / 2}\right)=-\left(2-2^{1 / 2}\right) \Phi(0)^{1 / 2} .
$$

According to the Schwarz inequality, the second integral

$$
\int_{0}^{\tau}(-\dot{\mathcal{U}})^{1 / 2} d t \leq \tau^{1 / 2}\left(\int_{0}^{\tau}(-\dot{\mathcal{U}}) d t\right)^{1 / 2}=\tau^{1 / 2}(\mathcal{U}(0)-\mathcal{U}(\tau))^{1 / 2} .
$$

However, $U(0)=\Phi(0)+T(0)=(1+\Delta) \Phi(0)$ and $U(\tau) \geq \Phi(\tau)=\frac{1}{2} \Phi(0)$ and, therefore,

$$
\int_{0}^{\tau}(-\dot{\mathcal{U}})^{1 / 2} d t \leq \tau^{1 / 2}\left(\frac{1}{2}+\Delta\right)^{1 / 2} \Phi(0)^{1 / 2}
$$


When we combine (4.5) with (4.6) and (4.7) we find the inequality

$$
\left(2-2^{1 / 2}\right) \Phi(0)^{1 / 2} \leq \tau^{1 / 2}\left(\frac{(\gamma-1) c \mu_{\max }(1+2 \Delta)}{k_{\min }}\right)^{1 / 2} \Phi(0)^{1 / 2}
$$

which, because $\Phi(0)>0$, implies the lower bound

$$
\tau \geq \frac{\left(6-4 \cdot 2^{1 / 2}\right) k_{\min }}{(\gamma-1) c \mu_{\max }(1+2 \Delta)} .
$$

This last is of the required form (4.2), for the constant

$$
A=\frac{\left(6-4 \cdot 2^{1 / 2}\right) k_{\min }}{c \mu_{\max }}
$$

is positive and depends upon $\mathbf{C}, c, \mathbf{K}$ only; indeed, $A$ depends upon $\mathbf{K}$ only through its symmetric part sym $\mathbf{K}$.

Lemma 1 demands no restriction upon $B$ beyond that it be a bounded regular region. We turn to showing that Lemma 1 can be made to furnish a lower bound on a half-life-and hence the conjecture framed in the Introduction remains correctunder less stringent boundary conditions on $\varphi$, provided the geometry of $B$ is restricted in a suitable way.

5. Parity. The parity transformation, or reflection in a fixed point (the origin say), maps the point $\mathbf{x}$ to the point $\overline{\mathbf{x}}=0-(\mathbf{x}-\mathbf{0})$. A point set is said to be parity-invariant if the transformation $\mathbf{x} \rightarrow \overline{\mathbf{x}}$ maps the set onto itself.

Any scalar, vector, or tensor field $f$, whose domain of definition is parity-invariant, can be resolved into the sum

$$
f=f^{+}+f^{-}
$$

of its even part $f^{+}$and its odd part $f^{-}$, where

$$
f^{+}(\mathbf{x})=\frac{1}{2}(f(\mathbf{x})+f(\overline{\mathbf{x}})), \quad f^{-}(\mathbf{x})=\frac{1}{2}(f(\mathbf{x})-f(\overline{\mathbf{x}})) .
$$

The even part remains invariant under the parity transformation, while the odd part transforms to its negative:

$$
f^{+}(\overline{\mathbf{x}})=f^{+}(\mathbf{x}), \quad f^{-}(\mathbf{x})=-f^{-}(\overline{\mathbf{x}}) .
$$

The key observation about the equations of thermoelasticity depends upon the assumption that $B$ and its boundary $\partial B$ are parity-invariant but, since the parityinvariance of $B$ implies that of $\partial B$, it is enough to make the assumption about $B$ alone.

LEMMA 2. Let $B$ be parity-invariant, and let $[\mathbf{u}, \mathbf{E}, \mathbf{S}, \varphi, \mathbf{g}, \mathbf{q}]$ be a thermoelastic process corresponding to zero body force and zero heat supply. Then

$$
\begin{aligned}
& {\left[\mathbf{u}^{+}, \mathbf{E}^{-}, \mathbf{S}^{-}, \varphi^{-}, \mathbf{g}^{+}, \mathbf{q}^{+}\right],} \\
& {\left[\mathbf{u}^{-}, \mathbf{E}^{+}, \mathbf{S}^{+}, \varphi^{+}, \mathbf{g}^{-}, \mathbf{q}^{-}\right],}
\end{aligned}
$$

are thermoelastic processes corresponding to zero body force and zero heat supply, and their respective surface tractions are

$$
\mathbf{s}^{+}=\mathbf{S}^{-} \mathbf{n}, \quad \mathbf{s}^{-}=\mathbf{S}^{+} \mathbf{n} .
$$


For the present purpose, it is the coupling of $\mathbf{u}^{+}$, the even part of the displacement, with $\varphi^{-}$, the odd part of the temperature difference, that is the significant feature.

The verification of the lemma is by means of a straightforward calculation. The even and odd parts of the spatial derivatives that appear in the underlying equations are

$$
\begin{aligned}
(\boldsymbol{\nabla u})^{+} & =\boldsymbol{\nabla}\left(\mathbf{u}^{-}\right), & (\boldsymbol{\nabla u})^{-} & =\boldsymbol{\nabla}\left(\mathbf{u}^{+}\right), \\
(\operatorname{div} \mathbf{S})^{+} & =\operatorname{div}\left(\mathbf{S}^{-}\right), & (\operatorname{div} \mathbf{S})^{-} & =\operatorname{div}\left(\mathbf{S}^{+}\right), \\
(\boldsymbol{\nabla} \varphi)^{+} & =\boldsymbol{\nabla}\left(\varphi^{-}\right), & (\boldsymbol{\nabla} \varphi)^{-} & =\boldsymbol{\nabla}\left(\varphi^{+}\right),
\end{aligned}
$$

whereas the even and odd parts of the temporal derivatives are

$$
\begin{array}{ll}
(\ddot{\mathbf{u}})^{+}=\left(\mathbf{u}^{+}\right)^{\cdot \cdot}, & (\ddot{\mathbf{u}})^{-}=\left(\mathbf{u}^{-}\right)^{\cdot \cdot}, \\
(\dot{\mathbf{E}})^{+}=\left(\mathbf{E}^{+}\right)^{\cdot}, & (\dot{\mathbf{E}})^{-}=\left(\mathbf{E}^{-}\right)^{\circ}, \\
(\dot{\varphi})^{+}=\left(\varphi^{+}\right)^{\circ}, & (\dot{\varphi})^{-}=\left(\varphi^{-}\right)^{\circ} .
\end{array}
$$

Thus, if we take the even part (of each side of) (2.1), the odd part of (2.2), the odd part of (2.3), the even part of (2.4), the odd part of (2.5), and the even part of (2.6), we obtain

$$
\begin{aligned}
\operatorname{div}\left(\mathbf{S}^{-}\right) & =\rho\left(\mathbf{u}^{+}\right)^{*}, \\
\operatorname{div}\left(\mathbf{q}^{+}\right) & =\rho c\left(\varphi^{-}\right)^{\cdot}+\theta_{0} \mathbf{M} \cdot\left(\mathbf{E}^{-}\right), \\
\mathbf{E}^{-} & =\operatorname{sym} \boldsymbol{\nabla}\left(\mathbf{u}^{+}\right), \\
\mathbf{q}^{+} & =\boldsymbol{\nabla}\left(\varphi^{-}\right) \\
\mathbf{S}^{-} & =\mathbf{C}\left[\mathbf{E}^{-}\right]-\varphi^{-} \mathbf{M}, \\
\mathbf{q}^{+} & =\mathbf{K} \mathbf{g}^{+} .
\end{aligned}
$$

Hence, $\left[\mathbf{u}^{+}, \mathbf{E}^{-}, \mathbf{S}^{-}, \varphi^{-}, \mathbf{g}^{+}, \mathbf{q}^{+}\right]$is a thermoelastic process corresponding to zero body force and zero heat supply, and, on repeating the argument with the roles of even and odd reversed, we find the same to be true of $\left[\mathbf{u}^{-}, \mathbf{E}^{+}, \mathbf{S}^{+}, \varphi^{+}, \mathbf{g}^{-}, \mathbf{q}^{-}\right]$.

In order to determine the associated surface traction, we use the fact that, when $B$ is parity-invariant, the normal at a boundary point $\mathbf{x}$ behaves according to the rule

$$
\mathbf{n}(\overline{\mathbf{x}})=-\mathbf{n}(\mathbf{x}) \text {. }
$$

Thus, $\mathbf{n}^{+}=\mathbf{0}$ and $\mathbf{n}=\mathbf{n}^{-}$. The surface traction is, therefore,

$$
\mathbf{s}=\mathbf{S n}=\left(\mathbf{S}^{+}+\mathbf{S}^{-}\right) \mathbf{n}^{-}=\mathbf{S}^{+} \mathbf{n}^{-}+\mathbf{S}^{-} \mathbf{n}^{-} .
$$

Under the parity transformation, the term $\mathbf{S}^{-} \mathbf{n}^{-}$remains invariant, being the product of two odd parts, but the term $\mathbf{S}^{+} \mathbf{n}^{-}$transforms to its negative. Thus, the even and odd parts of the surface traction are

$$
\mathbf{s}^{+}=\mathbf{S}^{-} \mathbf{n}^{-}=\mathbf{S}^{-} \mathbf{n}, \quad \mathbf{s}^{-}=\mathbf{S}^{+} \mathbf{n}^{-}=\mathbf{S}^{+} \mathbf{n},
$$

and the verification is complete.

6. Behaviour when $B$ is parity-invariant. If $B$ is parity-invariant it is possible to identify the separate parts of the mechanical energy that correspond to the even and odd parts of the displacement. Since $\operatorname{sym} \nabla\left(\mathbf{u}^{+}\right)=\mathbf{E}^{-}$and $\operatorname{sym} \nabla\left(\mathbf{u}^{-}\right)=\mathbf{E}^{+}$, the even part of the mechanical energy is

$$
\Phi^{+}=\frac{1}{2} \int_{B} \mathbf{E}^{-} \cdot \mathbf{C}\left[\mathbf{E}^{-}\right] d v+\frac{1}{2} \int_{B} \rho\left|\left(\mathbf{u}^{+}\right)^{\cdot}\right|^{2} d v,
$$


and the odd part is

$$
\boldsymbol{\Phi}^{-}=\frac{1}{2} \int_{B} \mathbf{E}^{+} \cdot \mathbf{C}\left[\mathbf{E}^{+}\right] d v+\frac{1}{2} \int_{B} \rho\left|\left(\mathbf{u}^{-}\right)^{\cdot}\right|^{2} d v .
$$

Because

$$
\Phi=\frac{1}{2} \int_{B}\left(\mathbf{E}^{+}+\mathbf{E}^{-}\right) \cdot \mathbf{C}\left[\mathbf{E}^{+}+\mathbf{E}^{-}\right] d v+\frac{1}{2} \int_{B} \rho\left|\left(\mathbf{u}^{+}\right)^{\cdot}+\left(\mathbf{u}^{-}\right)^{\cdot}\right|^{2} d v,
$$

and because

$$
\int_{B} \mathbf{E}^{+} \cdot \mathbf{C}\left[\mathbf{E}^{-}\right] d v=\int_{B} \mathbf{E}^{-} \cdot \mathbf{C}\left[\mathbf{E}^{+}\right] d v=\int_{B} \rho\left(\mathbf{u}^{+}\right)^{\cdot} \cdot\left(\mathbf{u}^{-}\right)^{\cdot} d v=0,
$$

as can be seen by making the change of variable $\mathbf{x} \rightarrow \overline{\mathbf{x}}$ in the multiple integrals, the mechanical energy decomposes into the sum of its even and odd parts:

$$
\Phi=\Phi^{+}+\Phi^{-} .
$$

The thermal energy likewise decomposes into the sum

$$
T=T^{+}+T^{-}
$$

of its even part $T^{+}$and its odd part $T^{-}$, where

$$
T^{+}=\frac{1}{2 \theta_{0}} \int_{B} \rho c\left(\varphi^{+}\right)^{2} d v, \quad T^{-}=\frac{1}{2 \theta_{0}} \int_{B} \rho c\left(\varphi^{-}\right)^{2} d v .
$$

Furthermore, the total energy decomposes into the sum

$$
U=\left(\Phi^{+}+T^{-}\right)+\left(\Phi^{-}+T^{+}\right)
$$

in which $\Phi^{+}+T^{-}$is the total energy associated with the thermoelastic process

$$
\left[\mathbf{u}^{+}, \mathbf{E}^{-}, \mathbf{S}^{-}, \varphi^{-}, \mathbf{g}^{+}, \mathbf{q}^{+}\right],
$$

and $\Phi^{-}+T^{+}$is the total energy associated with the thermoelastic process

$$
\left[\mathbf{u}^{-}, \mathbf{E}^{+}, \mathbf{S}^{+}, \varphi^{+}, \mathbf{g}^{-}, \mathbf{q}^{-}\right] \text {. }
$$

The even part of the mechanical energy has its own half-life $\tau^{+}$; this is the greatest lower bound of the set

$$
\left\{t: t \geq 0 \text { and } \Phi^{+}(t)=\frac{1}{2} \Phi^{+}(0)\right\}
$$

if the set is not empty, and is $+\infty$ otherwise. The odd part too has a half-life which can be defined in the obvious way, but we shall not need to do so.

It is now possible to proceed to our main result. We envisage a body occupying a region that is parity-invariant. The rate of working of the surface forces is arranged to be zero by subjecting a part of the boundary that is parity-invariant to a static displacement field, and leaving the remainder of the boundary traction-free. Furthermore the body is immersed in an environment whose temperature $\theta_{0}+\Theta(t)$ is spatially homogeneous at each instant $t$ but may vary with $t$ in any manner.

It might be hoped that it would be possible to take advantage of thermo-mechanical interaction, and to arrange the rapid damping out of a motion of the body by judicious control of the environmental temperature. What the result shows is that such an expectation must remain largely unfulfilled. 
Theorem. Let $B$ be parity-invariant, and let $[\mathbf{u}, \mathbf{E}, \mathbf{S}, \varphi, \mathbf{g}, \mathbf{q}]$ be a thermoelastic process corresponding to zero body force and zero heat supply which satisfies the boundary conditions

$$
\left.\dot{\mathbf{u}}\right|_{S_{1}}=\mathbf{0},\left.\quad \mathbf{s}\right|_{S_{2}}=\mathbf{0},\left.\quad \varphi\right|_{\partial B}=\Theta(t) \quad(t \geq 0),
$$

where $S_{1}$ and $S_{2}$ are complementary subsurfaces of $\partial B$ that are parity-invariant. Then the half-life

$$
\tau^{+} \geq \frac{A}{(\gamma-1)\left(1+2 T^{-}(0) / \Phi^{+}(0)\right)}
$$

provided $\Phi^{+}(0)>0$.

In summary, the original conjecture is confirmed in these circumstances, with the proviso that it now refers to the half-life of the even part of the mechanical energy.

It should be noted that one or the other of $S_{1}$ and $S_{2}$ may be empty.

The example of rapid decay constructed in Sec. 3 fails to meet the hypotheses of the theorem because $\mathbf{u}^{+}=\mathbf{0}$ and $\Phi^{+}=0$.

In order to prove the theorem, we consider the list $\left[\mathbf{u}^{+}, \mathbf{E}^{-}, \mathbf{S}^{-}, \varphi^{-}, \mathbf{g}^{+}, \mathbf{q}^{+}\right]$which, according to Lemma 2, is a thermoelastic process corresponding to zero body force and zero heat sopply. The associated surface traction is known to be $\mathbf{s}^{+}=\mathbf{S}^{-} \mathbf{n}$; the associated total energy is known to be $\Phi^{+}+T^{-}$; and the appropriate value of the ratio $\Delta$ is $T^{-}(0) / \Phi^{+}(0)$.

Because the subsurfaces $S_{1}$ and $S_{2}$ are parity-invariant, and because $(\dot{\mathbf{u}})^{+}=\left(\mathbf{u}^{+}\right)^{\cdot}$, the boundary conditions that $\mathbf{u}$ and $\mathbf{s}$ satisfy imply that

$$
\left.\left(\mathbf{u}^{+}\right)^{\cdot}\right|_{S_{1}}=\mathbf{0},\left.\quad \mathbf{s}^{+}\right|_{S_{2}}=\mathbf{0},
$$

and, hence, that

$$
\int_{B} \mathbf{s}^{+} \cdot\left(\mathbf{u}^{+}\right)^{\cdot} d a=0 \quad(t \geq 0) .
$$

Finally, the boundary condition on $\varphi$ implies that

$$
\left.\varphi^{-}\right|_{\partial B}=0 \quad(t \geq 0) \text {. }
$$

and the required lower bound on $\tau^{+}$now follows by an appeal to Lemma 1 .

\section{REFERENCES}

[1] C. M. Dafermos, On the existence and asymptotic stability of solutions to the equations of linear thermoelasticity, Arch. Rat. Mech. Anal. 29, 241-271 (1968)

[2] G. Dassios and M. Grillakis, Dissipation rates and partition of energy in thermoelasticity, Arch. Rat. Mech. Anal. 87, 49-91 (1984)

[3] W. A. Day, Energy decay in a problem of linear thermoelasticity, Mathematika 28, 102-115 (1981)

[4] M. E. Gurtin, The Linear Theory of Elasticity, Handbuch der Physik. Bd. VIa/2, Berlin, Springer, 1972

[5] D. E. Carlson, Linear Thermoelasticity, Handbuch der Physik. Bd. VIa/2, Berlin, Springer, 1972 